\title{
Research on Anti-Interference Nonlinear Adaptive Control Method for Autonomous Underwater Gliders
}

\author{
Wen-Qing Zhang, ${ }^{1,2}$ Liang-Long Da $\mathbb{C}^{1,2}$ De-Yan Tian, ${ }^{2}$ Qin-Dong Sun, ${ }^{1,2}$ \\ Wen-Long Wang, ${ }^{1,2}$ and Wen-Jing Chen ${ }^{2}$ \\ ${ }^{1}$ Naval Submarine Academy, Qingdao 266199, China \\ ${ }^{2}$ National Laboratory for Marine Science and Technology, Qingdao 266237, China \\ Correspondence should be addressed to Liang-Long Da; dalianglong@sohu.com
}

Received 5 March 2021; Revised 11 July 2021; Accepted 26 July 2021; Published 10 August 2021

Academic Editor: Xinggang Yan

Copyright (C) 2021 Wen-Qing Zhang et al. This is an open access article distributed under the Creative Commons Attribution License, which permits unrestricted use, distribution, and reproduction in any medium, provided the original work is properly cited.

In this paper, the characteristics of underactuation are analyzed according to the working principle of underwater glider, a complete 6DOF model of underwater glider is established, and the error equation of 3D path tracking is given. Based on the lineof-sight navigation method in three-dimensional space, the nonlinear adaptive control strategy is designed, and the roll angle control law, pitch angle control law, and depth control law of underwater glider are given. The tracking error of underwater glider is proved to be convergent by Lyapunov stability theorem. Finally, the results of marine experiments verify that the nonlinear controller designed in this paper can overcome the influence of constant current disturbance under certain conditions and has good robustness and good tracking effect under different ocean current sizes and course angles. Of course, one control method cannot be applicable to all sea conditions. When the angle between the size and direction of the ocean current exceeds a certain value, the tracking control of the underwater glider will show obvious divergence. Therefore, the stability range against the interference of the sea current is given in this paper.

\section{Introduction}

Underwater gliders have the advantages of long endurance and low energy consumption and can be applied to a variety of complex sea conditions. More and more underwater unmanned mobile platforms such as underwater gliders, wave gliders, and underwater unmanned vehicles are used to carry out tasks. Research on nonlinear and underactuated characteristics of underwater unmanned mobile platform and related control methods has become a hot topic in the control field $[1,2]$.

In recent years, the working mode of autonomous ocean vehicles has developed from the single operation mode to the cooperative formation operation mode [3]. Multiautonomous ocean vehicles can cooperate with each other to accomplish tasks that a single individual cannot accomplish. Therefore, the research on cooperative path tracking control of multiple autonomous marine vehicles has become a hot spot. In Dan Wang's doctoral thesis [4] of Dalian Maritime
University, aiming at the problem of cooperative path tracking control about autonomous marine vehicles with uncertainties and environmental disturbances, combined with dynamic surface control technology, the cooperative path tracking controllers of fully driven autonomous marine vehicles and underactuated autonomous marine vehicles are designed, respectively. A cooperative path tracking control strategy based on the adaptive dynamic surface method is proposed. Simulation results show the effectiveness of the proposed control algorithm. Zhang studied the cooperative tracking control of multiple UUVs to a given path under the constraint of master-slave formation. Based on the detailed analysis and summary of Lyapunov system theory and backstepping design theory, combined with the master-slave formation strategy, the cooperative formation strategy of master-slave multiunmanned underwater vehicles is designed according to the idea that UUV tracking error finally converges to zero. The cooperative tracking of the given desired path by multiple unmanned underwater 
vehicles under the constraint of master-slave formation is realized. And the designed cooperative path tracking controller is simulated and verified [5]. To solve the problem of 3D path tracking control for underactuated unmanned underwater vehicles, Hongjian et al. [6] designed a linear control term stabilized position tracking system in the form of tracking error feedback gain. Then, a dynamic controller is designed based on the backstepping method, which eliminates some nonlinear terms, simplifies the form of virtual control variables, and ensures the consistent and ultimately bounded state of the closed-loop tracking error system.

There are many research studies on unmanned platforms with strong AUV maneuverability in domestic and foreign literatures [7-10], considering the essential difference between the underactuation of underwater gliders and AUV [11-15]. In this paper, a mathematical model of underactuated underwater glider is established and a nonlinear controller is designed under the condition of ocean current interference. Based on the line-of-sight navigation algorithm, the expected pitch angle and yaw angle are designed, and the axial thrust, pitch moment, and yaw moment are obtained. A path tracking strategy [16-18] for the underactuated autonomous underwater vehicle is proposed.

\section{Underwater Glider Model Building}

The structure composition of the underwater glider platform $[19,20]$ is shown in Figure 1. The glider's workflow can be divided into four stages [21, 22]: surface preparation, gliding down, gliding up, and waiting on the surface. At the beginning of its voyage, an underwater glider floats on the surface of the water under the effect of net buoyancy. After receiving the control command through satellite communication or wireless communication on deck, the glider relies on the buoyancy regulating unit to return oil from the external oil sac to the internal oil cylinder, which reduces the drainage volume of the glider itself and makes the buoyancy less than gravity, thus sinking. At the same time, the attitude adjustment mechanism changes the position of the center of gravity by moving the internal weight so that the glider can reach the desired attitude angle. During the dive, it glides forward and down, using hydrodynamic forces on its horizontal and vertical fins, while mission sensors measure data. After reaching the predetermined working depth, the glider drains the oil from the internal cylinder to the external oil bag through the buoyancy adjusting unit, which increases the glider's own drainage volume and makes the buoyancy greater than gravity, thus realizing the transformation of the system movement from descending to ascending process. At the same time, the attitude adjustment mechanism also works to make it reach the attitude angle required for upward glide. During the gliding process [23, 24], the underwater glider adjusts its attitude in real time through the attitude adjustment mechanism and carries out stable gliding movement according to the set gliding angle and heading angle. If the glider needs to turn, the position of the center of gravity of the platform can be changed by the attitude adjustment mechanism, and the course angle can be deflected by the roll torque and hydrodynamic torque. After completing the task and returning to the water surface $[25,26]$, the underwater glider will make the communication antenna reach out of the water according to the preset program, carry out satellite positioning, and transmit the measured data to the control center through the satellite. At the same time, it will receive new control instructions and carry out the next cycle of work.

The six-degree-of-freedom (6DOF) mathematical model of the underactuated underwater glider [27] is as follows:

$$
\begin{aligned}
& \dot{X}=J(\Omega) v, \\
& M \dot{v}+C(v) v+D(v) v+g(X)=\tau, \\
& J(\Omega)=\left[\begin{array}{cc}
J_{1}(\Omega) & O_{3 \times 3} \\
O_{3 \times 3} & J_{2}(\Omega)
\end{array}\right], \quad J_{2}(\Omega)=
\end{aligned}
$$

where

$\left[\begin{array}{ccc}1 & \sin \varphi \tan \theta & \cos \varphi \tan \theta\end{array}\right]$

$\left[\begin{array}{ccc}1 & \cos \varphi & -\sin \varphi \\ 0 & \sin \varphi / \cos \theta & \cos \varphi / \cos \theta\end{array}\right]$, and $J_{1}(\Omega)=\left[\begin{array}{ll}\cos \psi & \cos \end{array}\right.$ $\theta 0 \sin \theta \sin \varphi-\sin \psi \cos \varphi \operatorname{sos} \psi \sin \theta \cos \varphi+\sin \psi$ $\sin \varphi \sin \psi \cos \theta \sin \psi \quad \sin \theta \sin \varphi+\cos \psi \cos \phi i n \psi \sin$ $\theta \cos \varphi-\cos \quad \psi \sin \varphi-\sin \theta \operatorname{os} \theta \sin \varphi \operatorname{os} \theta \cos \varphi$ ]. $\quad X=$ $(x, y, z, \varphi, \theta, \psi)^{T}$ and $\Omega=(\varphi, \theta, \psi)^{T}$ are the position and angle in inertial coordinate system, and $v=(u, v, w, p, q, r)^{T}$ indicates the speed in the moving coordinate system and meets the following conditions:

$$
\left\{\begin{array}{l}
M=M^{T}>0, \quad \dot{M}=0, \\
C(v)=-C^{T}(v), \quad X^{T} C(v) X=0, \\
X^{T} D(v) X>0, \quad \forall v \in R^{6}, \forall X \in R^{6} \backslash\{0\} .
\end{array}\right.
$$

The design goal of the control system in this paper is to design a feedback control law for the buoyancy, rolling moment, and pitching moment of the oil bag under the condition of unknown constant current interference and uncertain model parameters so that AUG position $(x, y, z)$ can accurately track the desired trajectory $\left(x_{d}, y_{d}, z_{d}\right)$, and all closed-loop system state variables are bounded. Figure 2 is the principle scheme of nonlinear control of underactuated underwater glider.

\section{Nonlinear Controller Design}

Define tracking error as follows:

$$
e=\left(e_{1}, e_{2}, e_{3}\right)^{T}=Q^{T}\left(X-X_{d}\right),
$$

where $X=(x(t), y(t), z(t))^{T} \in R^{3}$ indicates the actual position of AUG, $X_{d}=\left(x_{d}(s), y_{d}(s), z_{d}(s)\right)^{T} \in R^{3}$ is the desired path, and $\gamma_{d}(s)=\arctan y_{d}(s) / x_{d}(s)$.

$$
\begin{aligned}
\rho_{d}(s) & =\arctan \frac{-z_{d}(s)}{\sqrt{x_{d}^{2}(s)+y_{d}^{2}(s)}}, \\
Q & =\left[\begin{array}{ccc}
\cos \gamma_{d} \cos \rho_{d} & -\sin \gamma_{d} & \cos \gamma_{d} \sin \rho_{d} \\
\sin \gamma_{d} \cos \rho_{d} & \cos \gamma_{d} & \sin \gamma_{d} \sin \rho_{d} \\
-\sin \rho_{d} & 0 & \cos \rho_{d}
\end{array}\right],
\end{aligned}
$$




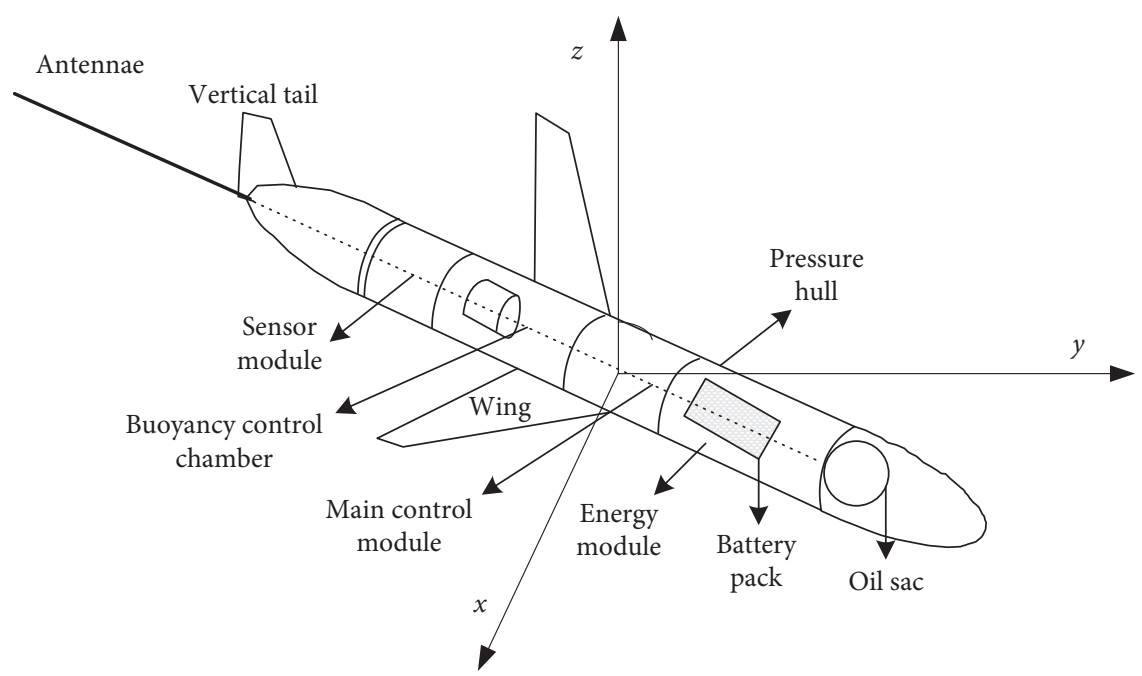

Figure 1: Schematic diagram of underwater glider.

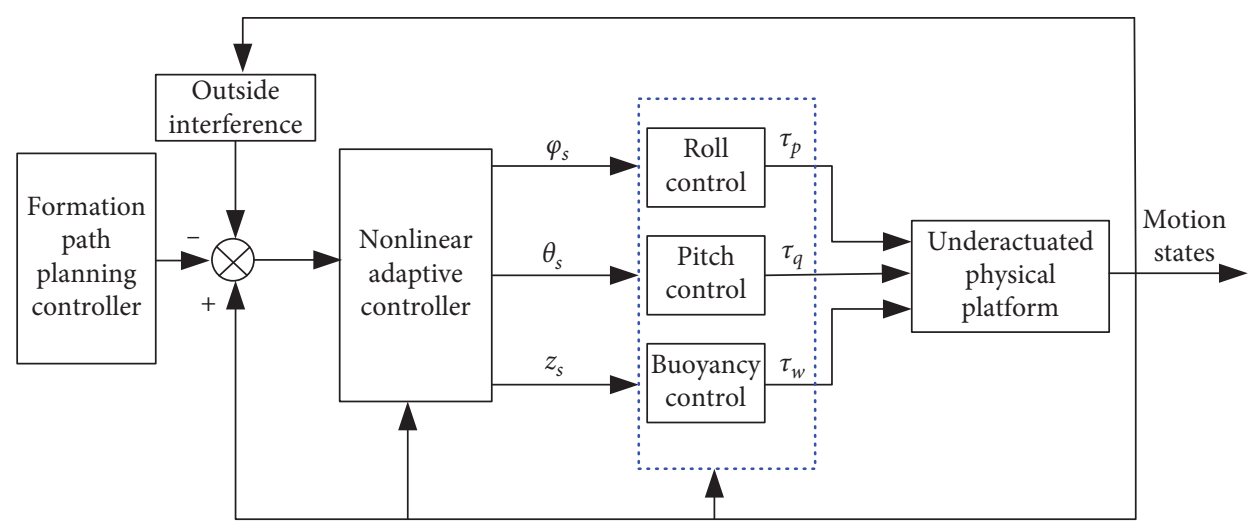

Figure 2: Principle scheme of nonlinear control of underactuated AUG.

where $s$ is the expected path operator, and the corresponding relationship of the above symbols is as follows:

$$
\begin{aligned}
& x_{d}^{\prime}(s)=\frac{\mathrm{d} x_{d}}{\mathrm{~d} s}, \\
& y_{d}^{\prime}(s)=\frac{\mathrm{d} y_{d}}{\mathrm{~d} s}, \\
& z_{d}^{\prime}(s)=\frac{\mathrm{d} z_{d}}{\mathrm{~d} s}, \\
& \dot{x}_{d}(s)=\frac{\partial x_{d}}{\partial s} \dot{s}, \\
& \dot{y}_{d}(s)=\frac{\partial y_{d}}{\partial s} \dot{s}, \\
& \dot{z}_{d}(s)=\frac{\partial z_{d}}{\partial s} \dot{s} .
\end{aligned}
$$

Define $\left\{X_{d}, Y_{d}, Z_{d}\right\}$ as the desired path coordinate system; let $\rho_{r}$ represent the angle between plane $\left\{X_{d}, Y_{d}\right\}$ and AUG actual speed $\dot{X}$ and $\gamma_{r}$ represent the angle between coordinate axes $X_{d}$ to $\dot{X}$ projected on plane $\left\{X_{d}, Y_{d}\right\}$.
Then, the position error dynamics are

$$
\left\{\begin{array}{l}
\dot{e}_{1}=e_{2} \dot{\gamma}_{d} \cos \rho_{d}-e_{3} \dot{\rho}_{d}+H \cos \gamma_{r} \cos \rho_{r}-H_{d}, \\
\dot{e}_{2}=-e_{1} \dot{\gamma}_{d} \cos \rho_{d}-e_{3} \dot{\gamma}_{d} \sin \rho_{d}+H \sin \gamma_{r} \cos \rho_{d}, \\
\dot{e}_{3}=e_{1} \dot{\rho}_{d}+e_{2} \dot{\gamma}_{d} \sin \rho_{d}-H \sin \rho_{d},
\end{array}\right.
$$
where $H_{d}=\sqrt{\dot{x}_{d}^{2}+\dot{y}_{d}^{2}+\dot{z}_{d}^{2}}$ and $H=\sqrt{\dot{x}^{2}+\dot{y}^{2}+\dot{z}^{2}}$.

Firstly, according to the three-dimensional LOS line-ofsight navigation algorithm, we get

$$
\begin{aligned}
\dot{s} & =\frac{H \cos \gamma_{r} \cos \rho_{r}+\delta_{1} e_{1}}{\sqrt{x_{d}^{\prime 2}+y_{d}^{\prime 2}+z_{d}^{\prime 2}}}, \\
\gamma_{r} & =\arctan -\frac{e_{2}}{\Delta_{1}}, \\
v_{r} & =\arctan \frac{e_{3}}{\Delta_{2}},
\end{aligned}
$$

where $\Delta_{1}>0, \Delta_{2}=\delta_{2} \sqrt{e_{2}^{2}+\Delta_{1}^{2}}>0, \delta_{1}>0$, and $\delta_{2}>0$ are the design parameters. 


$$
\begin{aligned}
& v_{\delta}=\arcsin \left(\sin \rho_{d} \cos \rho_{r} \cos \gamma_{r}+\cos \rho_{d} \sin \rho_{r}\right) \\
& \gamma_{\delta}=\arctan \left(\frac{\cos \gamma_{d} \sin \gamma_{r} \cos \rho_{r}-\sin \rho_{d} \sin \rho_{r} \sin \gamma_{d}+\sin \gamma_{d} \cos \gamma_{r} \cos \rho_{d} \cos \rho_{r}}{-\sin \gamma_{d} \sin \gamma_{r} \cos \rho_{r}-\sin \rho_{d} \sin \rho_{r} \cos \gamma_{d}+\cos \gamma_{d} \cos \gamma_{r} \cos \rho_{d} \cos \rho_{r}}\right) .
\end{aligned}
$$

It can be known that the desired pitch angle and yaw angle are as follows:

$$
\begin{aligned}
& \theta_{\text {ref }}=v_{\delta}-\arctan \frac{-w}{\sqrt{u^{2}+v^{2}}}, \\
& \psi_{\text {ref }}=\gamma_{\delta}-\arctan \frac{v}{u} .
\end{aligned}
$$

Let $\left(w_{s}, \theta_{s}, \psi_{s}\right)$ be the filtered expected value of $\left(w_{\text {ref }}, \theta_{\text {ref }}, \psi_{\text {ref }}\right)$. Next, design actually controls the buoyancy $\tau_{w}$, rolling moment $\tau_{p}$, and pitching moment $\tau_{q}$ of the input oil bladder.

Define

$$
\begin{aligned}
& K_{1}=\left[\begin{array}{ll}
k_{11} & k_{12}
\end{array}\right]^{T}=\left[\begin{array}{ll}
\varphi-\varphi_{s} & \theta-\theta_{s}
\end{array}\right]^{T}, \\
& K_{2}=v-a=\left[\begin{array}{lll}
u-a_{1} & v-a_{2} & w-a_{3} \\
p-a_{4} & q-a_{5} & r-a_{6}
\end{array}\right]^{T} .
\end{aligned}
$$

The virtual input is designed as follows:

$$
\begin{aligned}
& a_{3}=w_{s}, \\
& a_{4}=\cos \varphi\left(\dot{\varphi}_{s}-k_{11}\right)+\sin \varphi \cos \theta\left(\dot{\theta}_{s}-k_{12}\right), \\
& a_{5}=-\sin \varphi\left(\dot{\varphi}_{s}-k_{11}\right)+\cos \varphi \cos \theta\left(\dot{\theta}_{s}-k_{12}\right) .
\end{aligned}
$$

Take the first derivative of both sides of equations (12) and (13) to obtain

$$
\begin{aligned}
\dot{K}_{1} & =\left[\begin{array}{ll}
\dot{k}_{11} & \dot{k}_{12}
\end{array}\right]^{T}=\left[\begin{array}{ll}
\dot{\varphi}-\dot{\varphi}_{s} & \dot{\theta}-\dot{\theta}_{s}
\end{array}\right]^{T} \\
\dot{K}_{2} & =J_{3}\left[\begin{array}{ll}
p & q
\end{array}\right]^{T}-\left[\begin{array}{ll}
\dot{\varphi}_{s} & \dot{\theta}_{s}
\end{array}\right]^{T} \\
& =J_{3} G\left(K_{2}+a\right)-\left[\begin{array}{ll}
\dot{\varphi}_{s} & \dot{\theta}_{s}
\end{array}\right]^{T}
\end{aligned}
$$

where $\quad J_{3}=\left[\begin{array}{cc}\cos \varphi & -\sin \varphi \\ \sin \varphi / \cos \theta & \cos \varphi / \cos \theta\end{array}\right]$ and $\quad G=$ $\left[\begin{array}{llllll}0 & 0 & 0 & 1 & 0 & 0 \\ 0 & 0 & 0 & 0 & 1 & 0\end{array}\right]$

Then, we can get

$$
\dot{K}_{1}=-K_{1}+J_{3} G K_{2} .
$$

Find the first derivative of both sides and combine the dynamic equation of the AUG model to get

$$
\begin{aligned}
M \dot{K}_{2} & =M(\dot{v}-\dot{a}) \\
& =\tau-C(v)\left(K_{2}+a\right)-D(v)\left(K_{2}+a\right)-g(X)-M \dot{a} \\
& =\tau-(C(v)+D(v)) K_{2}-(C(v)+D(v)) a-g(X)-M \dot{a} .
\end{aligned}
$$

The design actual control input is

$$
\begin{aligned}
\tau_{w}= & -b_{3} k_{23}+\left(M-X_{\dot{u}}\right) \dot{a}_{1}+\left(M-Z_{\dot{w}}\right) w a_{5} \\
& +\left(Y_{\dot{v}}-M\right) v a_{6}+\left(X_{u}+X_{u|u|}|u|\right) a_{1}, \\
\tau_{p}= & -b_{4} k_{24}-k_{11} \cos \varphi-k_{12} \sin \varphi \cos \theta \\
& +\left(I_{y}-M_{\dot{q}}\right) \dot{a}_{5}+\left(Z_{\dot{w}}-M\right) w a_{1}+\left(M-X_{\dot{u}}\right) u a_{3} \\
& +\left(N_{\dot{r}}-I_{z}\right) r a_{4}+\left(I_{x}-K_{\dot{p}}\right) p a_{6}+\left(M_{q}+M_{q|q|}|q|\right) a_{5}, \\
\tau_{q}= & -b_{5} k_{25}+k_{11} \sin \varphi-k_{12} \cos \varphi \cos \theta \\
& +\left(I_{z}-N_{\dot{r}}\right) \dot{a}_{6}+\left(Y_{\dot{v}}-M\right) v a_{1}+\left(X_{\dot{u}}-M\right) u a_{2} \\
& +\left(I_{y}-M_{\dot{q}}\right) q a_{4}+\left(K_{\dot{p}}-I_{x}\right) p a_{5}+\left(N_{r}+N_{r|r|}|r|\right) a_{6} .
\end{aligned}
$$

\section{Stability Analysis}

Theorem 1. Assuming that the desired path is smooth and satisfies $\eta_{d}, \dot{\eta}_{d} \in L_{\infty}$ and $\Omega_{d}, \dot{\Omega}_{d}, \ddot{\Omega}_{d} \in L_{\infty}$, using the controller designed in this paper, the tracking error $\left(e_{1}, e_{2}, e_{3}\right)$ will eventually tend to zero; all signals in equation $V_{1}$ below are uniformly and ultimately bounded.

To prove: the Lyapunov function [28] is defined as follows:

$$
V_{1}=\frac{1}{2} K_{1}^{T} B_{1} K_{1}+\frac{1}{2} K_{2}^{T} M K_{2}+\frac{1}{2} \sum_{i=1}^{3} \Omega_{i}^{T} \Gamma_{i}^{-1} \widetilde{\Omega}_{i}+\widetilde{X}_{1}^{T} P \widetilde{X}_{1},
$$

where $\mathrm{B}_{1}=\operatorname{diag}\left\{b_{1}, b_{2}\right\}>0$ is the control parameter, and $\Gamma_{i}=\operatorname{diag}\left(\gamma_{i j}\right), \widetilde{X}_{1}=\left[\begin{array}{ll}\widetilde{v} & \widetilde{\eta}\end{array}\right]^{T}$.

Derive the two sides of the above formula, substitute them into formulas (16), (17), (21), and (25), and sort them out as follows:

$$
\begin{gathered}
\dot{V}_{1}=K_{1}^{T} B_{1} \dot{K}_{1}+K_{2}^{T} M \dot{K}_{2}+\sum_{i=1}^{3} \Omega_{i}^{T} \Gamma_{i}^{-1} \dot{\widetilde{\Omega}}_{i} \\
\quad \dot{\tilde{X}}_{1}^{T} P \widetilde{X}_{1}+\widetilde{X}_{1}^{T} P \dot{\widetilde{X}}_{1} . \\
\text { Let } A=\left[\begin{array}{cc}
-K_{3} & I \\
-K_{4} & 0
\end{array}\right], \\
\dot{V}_{1}=-K_{1}^{T} B_{1} K_{1}-K_{2}^{T} D K_{2}-K_{2}^{T} C K_{2} \\
+K_{2}^{T}\left(G^{T} J_{2}^{T} Z_{1} B_{1}+\tau-g-D a+D v-C a+C v-M \dot{a}\right) \\
+\sum_{i=1}^{3} \Omega_{i}^{T} \Gamma_{i}^{-1} \dot{\widetilde{\Omega}}_{i}+\dot{\widetilde{X}}_{1}^{T} A^{T} P \widetilde{X}_{1}+\widetilde{X}_{1}^{T} P A \dot{\widetilde{X}}_{1} .
\end{gathered}
$$

According to formula (2), $K_{2}^{T} C K_{2}=0$.

Combined with formula (18), there is a positive definite matrix $P$ so that $A^{T} P+P A=-Q$, where $Q$ is a Hermite matrix, so that 


$$
\begin{aligned}
\dot{V}_{1} \leq & -K_{1}^{T} B_{1} K_{1}-K_{2}^{T}\left(D+B_{2}\right) K_{2}-\widetilde{X}_{1}^{T} Q \widetilde{X}_{1} \\
\leq & -\lambda_{\min }\left(B_{1}\right)\left\|K_{1}\right\|^{2}-\lambda_{\min }\left(D+B_{2}\right)\left\|K_{2}\right\|^{2} \\
& -\lambda_{\min }(Q)\left\|\widetilde{X}_{1}\right\|^{2} .
\end{aligned}
$$

Then,

$$
\dot{V}_{1} \leq-\mu V_{1}+\delta
$$

where

$$
\begin{aligned}
B_{2} & =\operatorname{diag}\left\{\begin{array}{llllll}
b_{1} & b_{2} & b_{3} & b_{4} & b_{5} & b_{6}
\end{array}\right\}, \\
\mu & =\min \left(1,2 \lambda_{\min }\left(B_{1}\right), \frac{2 \lambda_{\min }\left(D+B_{2}\right)}{\lambda_{\max }(M)}, \frac{\lambda_{\min }(Q)}{\lambda_{\max }(P)}\right), \\
\delta & =\frac{1}{2} \sum_{i=1}^{3} \widetilde{\Omega}_{i}^{T} \Gamma_{i}^{-1} \widetilde{\Omega}_{i}, \\
V_{1}(t) & \leq V_{1}\left(t_{0}\right) e^{-\mu\left(t-t_{0}\right)}+\frac{\delta}{\mu} .
\end{aligned}
$$

Furtherly, we get

$$
\begin{aligned}
& \min \left(\frac{1}{2}, \frac{1}{2} \lambda_{\min }(M), \frac{1}{2} \gamma_{i j}^{-1}, \lambda_{\min }(P)\|X(t)\|^{2}\right) \\
& \leq\left\|X^{*}\left(t_{0}\right)\right\|^{2} \max \left(\begin{array}{c}
\frac{1}{2}, \frac{1}{2} \lambda_{\min }(M), \frac{1}{2} \gamma_{1 j}^{-1}, \\
\frac{1}{2} \gamma_{2 j}^{-1}, \frac{1}{2} \gamma_{3 j}^{-1}, \lambda_{\min }(P)
\end{array}\right) e^{-\mu\left(t-t_{0}\right)} \\
& \quad+\frac{\delta}{\mu},
\end{aligned}
$$

where $X(t)=\left[\begin{array}{llllll}Z_{1} & Z_{2} & \widetilde{\Omega}_{1} & \widetilde{\Omega}_{2} & \widetilde{\Omega}_{3} & \widetilde{X}_{1}\end{array}\right]^{T}$ and

$$
X^{*}\left(t_{0}\right)=\left[\begin{array}{c}
Z_{1}\left(t_{0}\right), Z_{2}\left(t_{0}\right), \widetilde{\Omega}_{1}^{T}\left(t_{0}\right), \\
\widetilde{\Omega}_{2}^{T}\left(t_{0}\right), \widetilde{\Omega}_{3}^{T}\left(t_{0}\right), \widetilde{X}_{1}\left(t_{0}\right)
\end{array}\right]^{T} .
$$

Define Lyapunov function as follows:

$$
\begin{aligned}
& V_{2}=\frac{1}{2} e_{1}^{2}+\frac{1}{2} e_{2}^{2}+\frac{1}{2} e_{3}^{2}, \\
& \dot{V}_{2}=e_{1} \dot{e}_{1}+e_{2} \dot{e}_{2}+e_{3} \dot{e}_{3} .
\end{aligned}
$$

Substitute into formulas (6) and (7) to get

$$
\begin{aligned}
\dot{V}_{2}= & -\delta_{1} e_{1}^{2}+e_{2} H \sin \left(\gamma-\gamma_{d}\right) \cos \left(\rho-\rho_{d}\right) \\
& -e_{3} H \sin \left(\rho-\rho_{d}\right) \\
= & -\delta_{1} e_{1}^{2}+e_{2} H \sin \left(k_{12}+\gamma_{r}\right) \cos \left(k_{11}+\rho_{r}\right) \\
& -e_{3} H \sin \left(k_{11}+\rho_{r}\right) .
\end{aligned}
$$

Substitute into formulas (8) and (9) and sort it out as follows:

$$
\begin{aligned}
& \dot{V}_{2}=-\delta_{1} e_{1}^{2}-\frac{\delta_{2} H}{\sqrt{\Delta_{2}^{2}+e_{3}^{2}}}\left(e_{2}^{2} \cos k_{12} \cos _{11}\right. \\
&-e_{2} \Delta_{1} \sin k_{12} \cos k_{11}-\frac{H}{\sqrt{\Delta_{2}^{2}+e_{3}^{2}} \sqrt{\Delta_{1}^{2}+e_{2}^{2}}} \\
&\left(e_{2} e_{3} \Delta_{1} \sin k_{12} \sin _{11}-e_{2}^{2} e_{3} \sin k_{11} \cos k_{12}\right) \\
& \quad-\frac{H}{\sqrt{\Delta_{2}^{2}+e_{3}^{2}}}\left(\Delta_{2} e_{3} \sin k_{11}+e_{3}^{2} \cos k_{11}\right) .
\end{aligned}
$$

As $\left|u_{d}\right| \geq\left|u_{d, \text { min }}\right|>0$, there is a finite time $t_{1} \geq t_{0} \geq 0$ which makes $H \geq H_{\text {min }}>0, \forall t \geq t_{1}$. And there is a finite timet $t_{2} \geq t_{1}$ which makes $\left|k_{1}\right| \leq\left|k_{1, s s}\right|$, where $\dot{V}_{2}\left(k_{1, s s}\right)<0$, defined on the interval $\left(t_{1}, t_{2}\right), \dot{V}_{2} \geq 0,\left(H, \cos k_{11}, \sin k_{11}\right.$, $\left.\sin k_{12}, \cos k_{12}\right) \in L_{\infty}$. Therefore, for $e_{1}\left(t_{0}\right) e_{2}\left(t_{0}\right) e_{3}\left(t_{0}\right)$ from any point, $e_{1}\left(t_{0}\right), e_{2}\left(t_{0}\right)$, and $e_{3}\left(t_{0}\right)$, when $t \longrightarrow \infty$, $\left(e_{1}, e_{2}, e_{3}\right) \longrightarrow 0$.

\section{Experimental Verification}

The physical parameters of the underwater glider platform in the numerical simulation experiments and sea trials are shown in Table 1 . The correctness and effectiveness of the path tracking control method studied above are verified.

5.1. Interference Experiments with Different Sea Conditions. The nonlinear adaptive control algorithm designed in this paper has been programmed into embedded program and loaded into the embedded control system of underwater glider. The embedded control system uses STM32F407 as the core processor, integrates FPU and DSP instructions, and the main frequency of the system is $72 \mathrm{MHz}$, which can carry out 32-bit floating point operation. C/OS-II real-time operating system is adopted, which can realize high speed operation and large capacity storage. Figure 3 shows the motion control test process of an underwater glider in the South China Sea.

Underwater glider test parameter setting: the coordinate system adopts the southeast geocoordinate system, the test time is $30 \mathrm{~h}$, and the control frequency is $10 \mathrm{~Hz}$; the path is designed as a straight line, the length of the path is about $30 \mathrm{~km}$, and the path node set is set as follows: $x_{d}=[0,5,10,15,20,25]$ and $y_{d}=[0,0,0,0,0,0]$, and the unit is $\mathrm{km}$. Single platform control parameters are as follows: roll angle control $k_{p}=1200, k_{i}=0$, and $k_{d}=-22$; trim angle control $k_{p}=220, k_{i}=10$, and $k_{d}=-30$; and depth control $k_{p}=1500, k_{i}=10$, and $k_{d}=-150$. Parameters of nonlinear adaptive control module are as follows: $K_{1}=I^{2 \times 2}, K_{2}=I^{6 \times 6}$, $\Delta_{1}=3.2, \delta_{1}=1.2$, and $\delta_{2}=2.0$, where $I$ is the identity matrix.

5.1.1. The Size of the Ocean Current is $1.0 \mathrm{kN}$, and It Runs under the Condition of $8^{\circ}$ Angle with the Direction of the Set Path. Under the condition that the path of the glider is a straight line, the size of the ocean current is $1.0 \mathrm{kN}$, and the 
TABLE 1: Lots of AUG model parameters.

\begin{tabular}{lc}
\hline Variables & Values \\
\hline$M$ & $69 \mathrm{~kg}$ \\
$m_{p}$ & $18 \mathrm{~kg}$ \\
$m_{r}$ & $18 \mathrm{~kg}$ \\
$L$ & $2.17 \mathrm{~m}$ \\
$I_{x}$ & $0.54 \mathrm{~kg} \cdot \mathrm{m}^{2}$ \\
$I_{y}$ & $19.75 \mathrm{~kg} \cdot \mathrm{m}^{2}$ \\
$I_{z}$ & $19.82 \mathrm{~kg} \cdot \mathrm{m}^{2}$ \\
$X_{\dot{u}}$ & $1.3 \mathrm{~kg}$ \\
$Y_{\dot{v}}$ & $79.66 \mathrm{~kg}$ \\
$Z_{\dot{w}}$ & $60.53 \mathrm{~kg}$ \\
$K_{\dot{p}}$ & $0 \mathrm{~kg} \cdot \mathrm{m}^{2}$ \\
$M_{\dot{q}}$ & $21.83 \mathrm{~kg} \cdot \mathrm{m}^{2}$ \\
$N_{\dot{n}}$ & $23.56 \mathrm{~kg} \cdot \mathrm{m}^{2}$ \\
$X_{u}$ & $-50 \mathrm{~kg} / \mathrm{s}$ \\
$Y_{v}$ & $-100 \mathrm{~kg} / \mathrm{s}$ \\
$Z_{w}$ & $-100 \mathrm{~kg} / \mathrm{s}$ \\
$Y_{v}$ & $-80 \mathrm{~kg} / \mathrm{s}$ \\
$Z_{w}$ & $-80 \mathrm{~kg} / \mathrm{s}$ \\
$K_{p}$ & $-20 \mathrm{~kg} \cdot \mathrm{m}^{2} / \mathrm{s}$ \\
$M_{q}$ & $-35 \mathrm{~kg} \cdot \mathrm{m}^{2} / \mathrm{s}$ \\
$N_{r}$ & $-35 \mathrm{~kg} \cdot \mathrm{m}^{2} / \mathrm{s}$ \\
$X_{u|u|}$ & $-12.37 \mathrm{~kg} / \mathrm{m}$ \\
$Y_{v|v|}$ & $19.35 \mathrm{~kg} / \mathrm{m}$ \\
$Z_{w|w|}$ & $0 \mathrm{~kg} / \mathrm{m}$ \\
$K_{p|p|}$ & $0 \mathrm{~kg} \cdot \mathrm{m}^{2}$ \\
$M_{q|q|}$ & $21.83 \mathrm{~kg} \cdot \mathrm{m}^{2}$ \\
$N_{r|r|}$ & $23.56 \mathrm{~kg} \cdot \mathrm{m}^{2}$ \\
\hline &
\end{tabular}

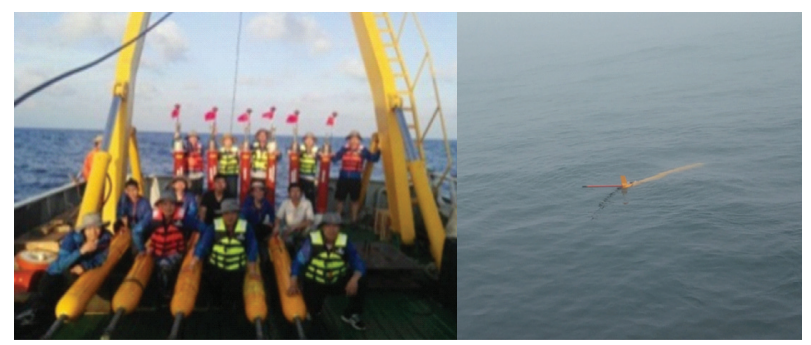

Figure 3: Underwater glider tests in the South China Sea.

angle between the direction of the ocean current and the direction of the path is $8^{\circ}$; the glider is operated by the nonlinear adaptive control method proposed in the paper.

Figure 4 shows the tracking process of the actual path and the expected path of the underwater glider. It can be seen from the figure that, in the case of unknown ocean current interference and unknown model parameters in the model, the controller can make the underwater glider track the expected trajectory stably, and the tracking error is within $10 \mathrm{~m}$, achieving the expected effect.

During the underwater glider test, the maximum gliding depth was set as $1000 \mathrm{~m}$. The gliding process of profile is shown in Figure 5. It can be seen that the underwater glider carried out repeated gliding movement at a depth of 0-1000 $\mathrm{m}$, and the depth control process was stable.

As shown in Figure 6, it can be seen that the trim angle of the underwater glider is stable tracking set value. The diving

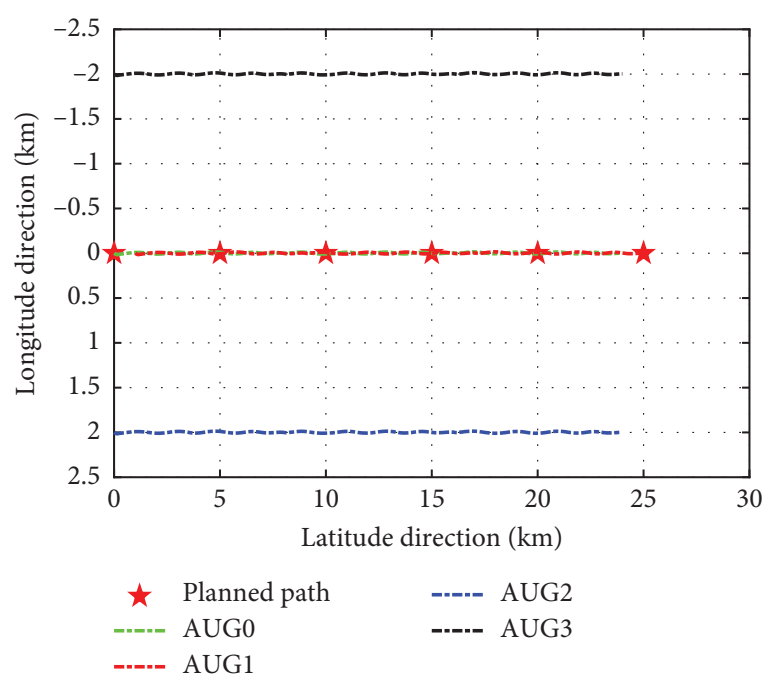

FIgURE 4: Stable running process of underwater glider tracking control.

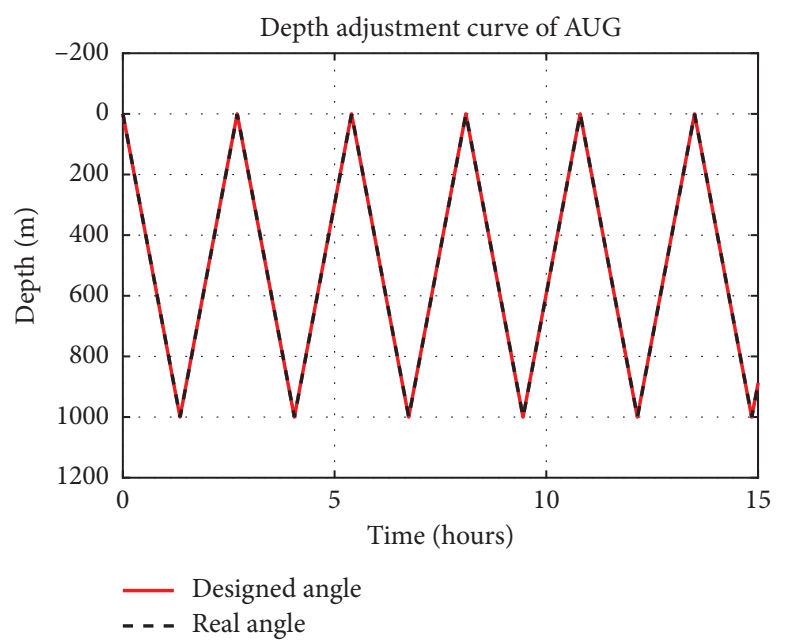

Figure 5: Depth profile control of underwater glider.

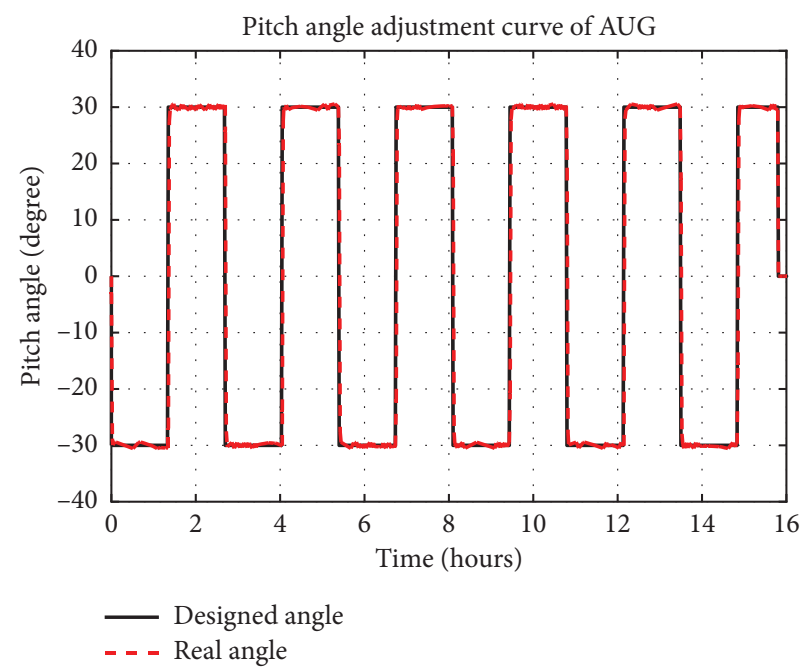

Figure 6: Trim angle control process of underwater glider. 
value is $-30^{\circ}$ and the floating value is $+30^{\circ}$, which achieves the expected effect.

As shown in Figure 7, it can be seen that the roll angle of the underwater glider is stable and tracks the set value, and the actual roll angle control achieves the desired effect.

5.1.2. The Size of the Ocean Current is $1.0 \mathrm{kN}$, and the Angle with the Path Direction is $10^{\circ}$. Under the condition that all test parameters and parameters of the glider remain unchanged, the size of the ocean current is set to be $1.0 \mathrm{kN}$ and the included angle with the path direction is $10^{\circ}$. The running results are shown in Figure 8. The tracking control of the glider is in a critical stable state with the tracking error within $50 \mathrm{~m}$.

\subsubsection{The Size of the Ocean Current is $1.0 \mathrm{kN}$, and the Angle} with the Path Direction is $12^{\circ}$. When all test parameters and parameters of the glider remain unchanged, the size of the ocean current is set at $1.0 \mathrm{kN}$ and the included angle with the path is $12^{\circ}$. The running results are shown in Figure 9, and the tracking control of the glider is gradually divergent.

5.2. Comparison Experiment of Control Effect. On the basis of the test in 5.1, all test parameters and control parameters of the underwater glider are kept unchanged. The control effect of the traditional PID control algorithm and the nonlinear adaptive control algorithm designed in this paper is compared.

5.2.1. Traditional Control Method. Single platform control parameters are as follows: roll angle control: $k_{p}=1200$, $k_{i}=0$, and $k_{d}=-22$; trim angle control: $k_{p}=220, k_{i}=10$, and $k_{d}=-30$; and depth control: $k_{p}=1500, k_{i}=10$, and $k_{d}=-150$. During the test, the expected yaw angle of the glider was set at $10^{\circ}$, the current size was $1.0 \mathrm{kN}$, and the included angle between the glider and the set heading was $8^{\circ}$. The test results are obtained in Figure 10. It can be found that the yaw angle of the underwater glider is controlled around $10^{\circ}$, and the trim angle has obvious oscillation within the range of up and down fluctuation of $2^{\circ}$. When the current amplitude decreases or the angle between the current direction and the set course decreases, the yaw angle control converges stably. When the current amplitude or the angle between the current direction and the set course increases, the divergence of yaw angle control will appear. Therefore, the stable range of the traditional control method against the ocean current interference at this time is within the size of the ocean current $1.0 \mathrm{kN}$ and within the angle of $8^{\circ}$ with the set course.

5.2.2. Nonlinear Adaptive Control Method. Single platform control parameters are as follows: roll angle control: $k_{p}=1200, k_{i}=0$, and $k_{d}=-22$; trim angle control: $k_{p}=220, k_{i}=10$, and $k_{d}=-30$; and depth control: $k_{p}=1500, k_{i}=10$, and $k_{d}=-150$. Parameters of nonlinear adaptive control module are as follows: $K_{1}=I^{2 \times 2}, K_{2}=I^{6 \times 6}$,

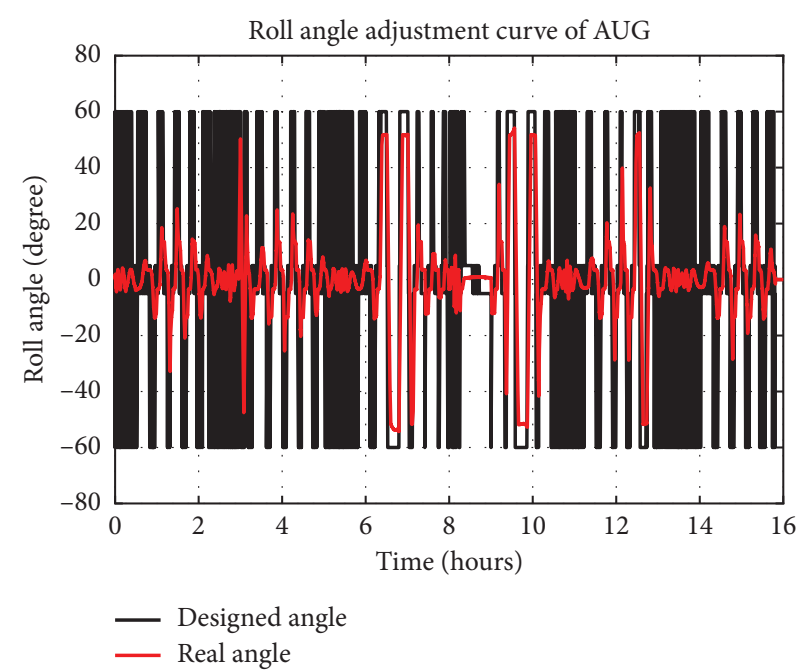

FIGURE 7: Roll angle control process of underwater glider.

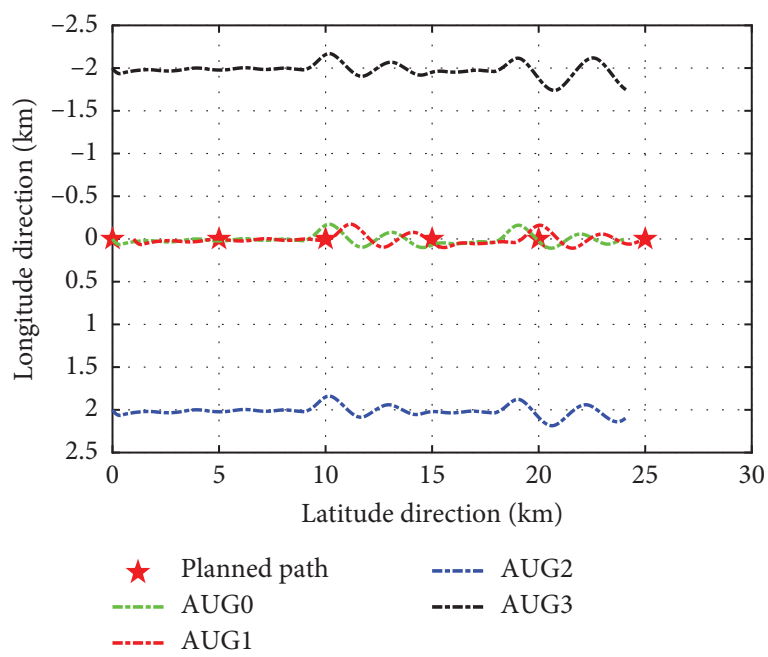

Figure 8: Critical stabilization process of underwater glider tracking control.

$\Delta_{1}=3.2, \delta_{1}=1.2$, and $\delta_{2}=2.0$, where $I$ is the identity matrix. The experimental conditions and ocean current disturbance are consistent with those of traditional methods. The yaw angle of the underwater glider was set as expected value of $10^{\circ}$, the current size was $1.0 \mathrm{kN}$, and the included angle with the set heading was $8^{\circ}$. The test results are obtained in Figure 11. It can be found that the yaw angle of the underwater glider is controlled in the vicinity of $10^{\circ}$ and within the range of fluctuation of $0.5^{\circ}$, which reaches the control expectation. When the current amplitude decreases or the angle between the current direction and the set course decreases, the yaw angle control converges stably. When the size of ocean current is $1.0 \mathrm{kN}$ and the angle between it and the set course is increased to $10^{\circ}$, the critical oscillation phenomenon will appear in the yaw angle control. Therefore, the stable range of the nonlinear adaptive control method designed in this paper against the ocean current interference is within $1.0 \mathrm{kN}$ of the ocean current size and within $10^{\circ}$ of 


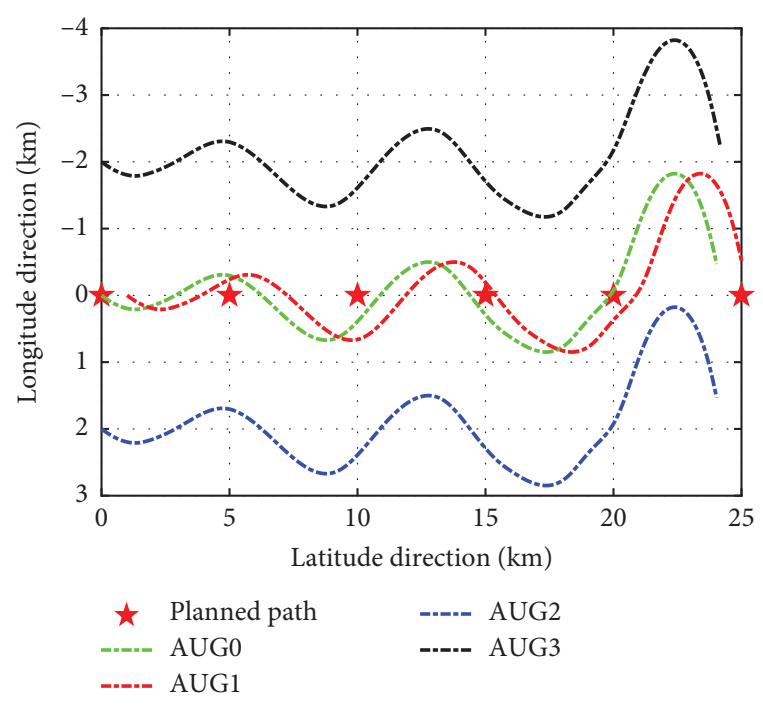

FIGURE 9: Divergence process of underwater glider tracking control.

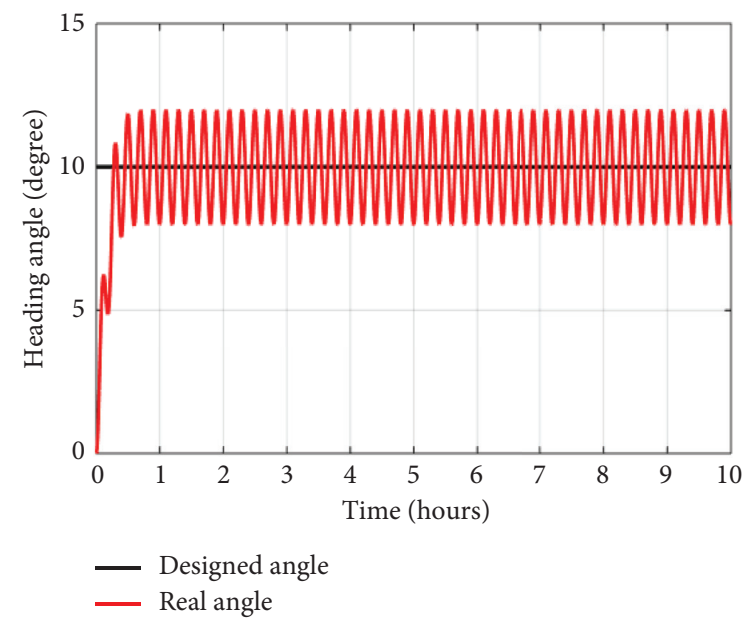

FIGURE 10: Pitch angle control process with the nonlinear active disturbance rejection control algorithm.

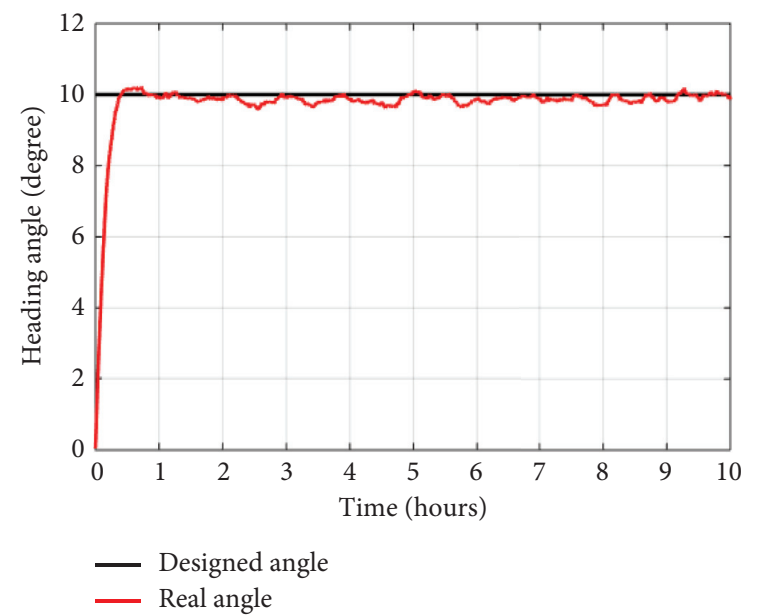

FIgURE 11: Pitch angle control process with the PID control algorithm.

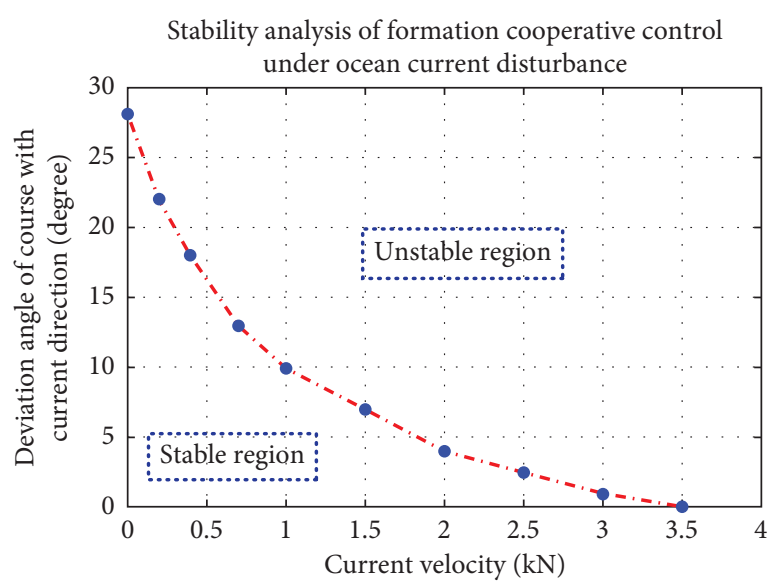

FIgURE 12: Stability analysis of formation cooperative control under ocean current disturbance.

the angle with the set course. The stability range of the nonlinear adaptive control method is larger than that of the traditional method, and the control effect is more obvious. However, for the step response index of the control algorithm, the steady-state running time is $0.5 \mathrm{~s}$. Since the control frequency of the underwater glider is slower than that of $10 \mathrm{~Hz}$, there is no obvious difference in the response time between the two control methods, and both of them can meet the dynamic course adjustment process of the underwater glider.

\subsubsection{Stable Range of Nonlinear Adaptive Control Method.} The nonlinear adaptive control method for underwater vehicle interference designed in this paper cannot be applied to all interference situations. Of course, there is no control algorithm that can be applied to all interference situations. The applicable scope of the control method of this paper is shown in Figure 12. Figure 12 shows the stability analysis of the formation of underwater gliders under the interference of ocean currents.

\section{Conclusions}

In this paper, the path tracking problem of underactuated underwater glider under the condition of ocean current interference is studied. Combined with the line-of-sight navigation method, an underactuated path tracking model of underwater glider is established and a nonlinear adaptive controller is designed. The roll angle control law, pitch angle control law, and depth control law of underwater glider are given. Through a large number of sea experiments, the control effect of underwater glider path following under different ocean current sizes and directions is analyzed. (1) Under the condition that the size of the ocean current is $1.0 \mathrm{kN}$ and the angle with the latitude direction is $8^{\circ}$, the tracking control of the underwater glider runs stably. (2) Under the condition that the ocean current size is $1.0 \mathrm{kN}$ and the angle with the latitude direction is $10^{\circ}$, the tracking control of the underwater glider is critical stable. (3) The tracking control of the glider gradually diverges under the 
condition that the ocean current size is $1.0 \mathrm{kN}$ and the angle with the latitude direction is $12^{\circ}$. (4) Compared with the traditional control algorithm, the nonlinear adaptive control algorithm designed in this paper has better control effect under the same sea conditions. It can be seen that when the size and direction of ocean current meet certain conditions, the controller proposed in this paper can overcome the influence of constant ocean current disturbance and unknown model parameters and has good robustness and good tracking effect. Of course, one control method cannot be applicable to all sea conditions. When the angle between ocean current size and direction exceeds a certain value, the tracking control of the underwater glider will show obvious divergence. Therefore, the stability range against ocean current interference is given in this paper (Figure 12).

\section{Data Availability}

The data used to support the findings of this study are included within the article.

\section{Conflicts of Interest}

The authors declare that there are no conflicts of interest.

\section{Authors' Contributions}

Wen-Qing Zhang carried out project demonstration and theoretical derivation; Liang-Long Da contributed to conceptualization; De-Yan Tian was responsible for software; Qin-Dong Sun reviewed and edited the manuscript; WenLong Wang designed the control system; Wen-Jing Chen designed the experimental plan. All authors have read and agreed to the final version of the manuscript.

\section{Acknowledgments}

This work was financially supported by National Key Research and Development Program of China (No. 2019YFC0311700) and Postdoctoral Research Fund of China (No. 2020T130771).

\section{References}

[1] W. Fang and W. Lei, "A survey on development of motion control for undemcmated AUG," Shipbuilding of China, vol. 51, no. 2, pp. 227-241, 2010.

[2] H. Bin, Research on Multi-AUV Formation Control and Cooperative Search Technology, Harbin Engineering University, Harbin, China, 2018.

[3] J. Heming, Z. Lijan, C. Xiangqin et al., "Three-dimensional path following control for an underactuated AUG based on nonlinear iterative sliding mode," Acta Automatica Sinica, vol. 38, no. 2, pp. 308-314, 2012.

[4] W. Dan, "Autonomous ocean vehicle cooperative path tracking based on adaptive dynamic surface control," Non Linear Dynamics, vol. 77, no. 1-2, 2014.

[5] Z. Qingyan, "Research on Multi-UUV Cooperative Path Tracking Control under Formation Constraints," Harbin Engineering University, Harbin, China, 2016.
[6] W. Hongjian, C. Ziyin, J. Heming et al., "Three-dimensional path-following control of underactuated unmanned underwater vehicle using feedback gain backstepping," Control Theory \& Applications, vol. 31, no. 1, pp. 66-77, 2014.

[7] G. Jian, X. Demin, Y. Weisheng, L. Mingyong, and Z. Fubin, "Applying cascaded systems theory to ensuring globally uniformly asymptotical stability of trajectory tracking controller of underactuated autonomous underwater vehicle (AUV)," Journal of Northwestern Polytechnical University, vol. 28, no. 3, pp. 404-408, 2010.

[8] W. Hongjian, C. Ziyin, J. Heming, and L. Juan, "Backstepping method with PID gain tuning in diving control of autonomous underwater vehicle," Control Theory \& Applications, vol. 29, no. 9, pp. 1139-1145, 2012.

[9] L. Zhicheng, "Research on underactuated UUV path following control based on adaptive sliding mode," Harbin Engineering University, Harbin, China, 2017.

[10] J. Heming, L. Zhang, X. Qi, and L. Yang, "Three-dimensional path tracking control for autonomous underwater vehicle based on neural network," Control Theory \& Applications, vol. 29, no. 7, pp. 877-883, 2012.

[11] Z. Nannan, Research on Path Planning and Path Tracking Methods of Underwater Robots, Jiangsu University of Science and Technology, Zhenjiang, China, 2019.

[12] J. Chunmeng, W. Lei, and S. Yushan, "Design of novel S-plane controller of autonomous underwater vehicle established on sliding mode control," Journal of Harbin Institute of Technology, vol. 24, no. 2, 2017.

[13] M. Santhakumar and K. Jinwhan, "Coordinated motion control in task space of an autonomous underwater vehiclemanipulator system," Ocean Engineering, vol. 104, pp. 155167, 2015.

[14] L. Wenbai, L. Mingyong, and L. Huxiong, "Positioning performance analysis of multi-AUV coordinated navigation system based on relative position measurement of single pilot," Acta Automatica Sinica, vol. 06, pp. 724-736, 2011.

[15] H. Hai, Z. Qiang, and Z. Shudi, "Under-driven AUV adaptive formation control strategy," Journal of Harbin Engineering University, vol. 05, pp. 633-637, 2015.

[16] X. Bo, B. Jinlei, and H. Yanling, "Research status and progress of multi-AUV collaborative navigation problem," Acta Automatica Sinica, vol. 03, pp. 445-467, 2015.

[17] J. M. Soares, A. P. Aguiar, A. M. Pascoal et al., "Joint ASV/ AUV range-based FormationControl: theory and experimental results," in International Inference on Robotics and Automation, pp. 5579-5585, IEEE, Karlsruhe, Germany, May 2013.

[18] J. B. De Sousa, J. Pereira, J. Pinto et al., "Rapid environmental picture Atlantic exercise 2015: A field report," in Proceedings of the Oceans, pp. 335-343, IEEE, Monterey, CA, United States, December 2016.

[19] L. Hao, H. Gu, F. Kang et al., "Virtual-leader based formation control with constant bearing guidance for underactuated AUVs," ICIC Express Letters, vol. 11, no. 1, pp. 117-125, 2017.

[20] L. C. Zhang, J. Wang, T. Wang et al., "Optimal formation of multiple AUVs cooperative localization based on virtual structure," in Proceedings of the Oceans, pp. 623-629, IEEE, Monterey, CA, United States, September 2016.

[21] M. Chen, H. Wang, and X. Liu, "Adaptive fuzzy practical fixed-time tracking control of nonlinear systems," IEEE Transactions on Fuzzy Systems, vol. 29, no. 3, pp. 664-673, 2021.

[22] H. Wang, W. Bai, X. Zhao et al., "Finite-Time-Prescribed performance-based adaptive fuzzy control for strict-feedback 
nonlinear systems with dynamic uncertainty and actuator faults," IEEE Transactions on Cybernetics, 2021.

[23] H. Zhou, Z. Wei, Z. Zeng et al., "Adaptive robust sliding mode control of autonomous underwater glider with input constraints for persistent virtual mooring," Applied Ocean Engineering, vol. 95, pp. 1-13, 2020.

[24] L. Zhang, X. Tao, and R. Zhang, "Motion control of autonomous underwater glider based on ISMC," in Proceedings of the Oceans 2018 MTS/IEEE, pp. 120-167, Charleston, SC,USA, October 2018.

[25] Z.-q. Su, M. Zhou, F.-f. Han et al., "Attitude control of underwater glider combined reinforcement learning with active disturbance rejection control," Journal of Marine Science and Technology, vol. 24, no. 3, pp. 686-704, 2019.

[26] J. Cao, J. Cao, Z. Zeng et al., "Nonlinear multiple-inputmultiple-output adaptive backstepping control of underwater glider systems," International Journal of Advanced Robotic Systems, pp. 1-14, 2016.

[27] H. Song, Y. Zhou, X. Sun et al., "Heading tracking control with an adaptive hybrid control for underactuated underwater glider," ISA Transactions, vol. 80, pp. 554-563, 2018.

[28] X. Sun, Y. Zhou, H. Sang et al., "Adaptive path following control for wave gliders in time-varing environment," Ocean Engineering, vol. 218, pp. 1-14, 2020. 